\title{
Magnetic Nanoparticles for Multi-Imaging and Drug Delivery
}

\author{
Jae-Hyun Lee, Ji-wook Kim, and Jinwoo Cheon*
}

\begin{abstract}
Various bio-medical applications of magnetic nanoparticles have been explored during the past few decades. As tools that hold great potential for advancing biological sciences, magnetic nanoparticles have been used as platform materials for enhanced magnetic resonance imaging (MRI) agents, biological separation and magnetic drug delivery systems, and magnetic hyperthermia treatment. Furthermore, approaches that integrate various imaging and bioactive moieties have been used in the design of multi-modality systems, which possess synergistically enhanced properties such as better imaging resolution and sensitivity, molecular recognition capabilities, stimulus responsive drug delivery with on-demand control, and spatio-temporally controlled cell signal activation. Below, recent studies that focus on the design and synthesis of multi-mode magnetic nanoparticles will be briefly reviewed and their potential applications in the imaging and therapy areas will be also discussed.
\end{abstract}

\section{INTRODUCTION}

Rapid advances made in nanotechnology have set the stage for potentially significant improvements in biotechnology applications that have the potential of enhancing public health and patient well being (Ferrari, 2005; Pankhurst et al., 2003; Peer et al., 2007; Sarikaya et al., 2003). Among the numerous nanomaterial based products developed thus far, inorganic nanoparticles constitute one of the most important platforms owing to their rigid characteristics and well-known surface tailoring capabilities (Cheon and Lee, 2008a; Kim et al., 2009; Wang, 2005). These types of nanoparticles, with sizes in the regime of 1-100 nm, display interesting materials properties never before observed for corresponding bulk-sized materials (Jana et al., 2004; Jun et al., 2008a; Kelly et al., 2003). For instance, a significant variation in electronic properties as a function of size is generally observed for semiconducting nanomaterials. Such properties can be used to tune light absorption characteristics in the ultraviolet to the near-infrared regions (Alivisatos, 1996; Dabbousi et al., 1997; Smith and Nie, 2010). Also, the frequencies of plasmon resonance, which results from free electron oscillation in-phase with an electromagnetic wave, change as the sizes or shapes of metallic nanomaterials are varied (Link and El-Sayed, 1999; Prodan et al., 2003).

Another important example of size-governed changes in properties is found in magnetic materials, which are the main theme of this review. Magnetic materials and especially ferromagnetic materials generally have permanent dipoles. However, in nanoscale regime, the permanent dipole disappears being replaced by a new magnetic property known as superparamagnetism. In the superparamagnetic regime, the net magnetic moment in a pool of nanoparticles is zero, even if the each single nanoparticle still possesses the dipole moment, because the orientations of dipole moments in each nanoparticle are randomly distributed by the thermal effect. Superparamagnetism and the resulting disappearance of a net magnetic moment needs to be avoided in magnetic storage devices (Bigioni et al., 2006; Moser et al., 2002; Sun, 2006). For example, the superparamagnetic limit governs construction of highly integrated hard disk drives where small sized magnetic grains are required. However, the fabrication of super-intergrated devices must take into account the fact that below a certain nanometer size small magnetic grains lose their magnetization.

Interestingly, superparamagnetism and the associated disappearance of a net magnetic moment is an important property for the use of magnetic nanoparticles in the biological and medical applications (Gupta and Gupta, 2005; Neuberger et al., 2005). Superparamagnetism enables nanoparticles injected into the blood stream to freely flow through micro vessels without suffering from magnetically induced clusterization, a phenomenon that can have harmful effects on the function of the cardiovascular system by causing embolic strokes. Owing to the development of various surface tailoring strategies, superparamagnetic nanoparticles are now widely used for various biological and medical purposes, such as agents for in vivo imaging and biomolecule detection/sensing, as well as vectors for drug delivery (Lee et al., 2007; Pankhurst et al., 2003). Below, the advantageous aspects of superparamagnetic nanoparticles for biomedical applications will be discussed and an emphasis will be given to recent advances made in their use in imaging and drug delivery systems, especially those that employ magnetic nanoparticles in multi-mode platforms. 


\section{MAGNETIC NANOPARTICLES AND SURFACE MODIFICATION}

At the current time, many types of magnetic nanoparticles with different sizes, shapes, and compositions are available. Also, the properties of the nanoparticles have been explored in order to uncover the physical source of nanoscale magnetism (Cullity, 1972; Jun et al., 2005; 2008a; Noh et al., 2012). Many methods have been developed thus far to synthesize magnetic nanoparticles. The hydrolytic co-precipitation method is most widely used because the required reaction conditions are readily scalable to an industrial level (Jung and Jacobs, 1995; Molday, 1984; Reimer et al., 1995). Briefly, in this technique reducing agents in the presence of appropriate surfactants are rapidly mixed with aqueous solutions of metallic precursors, such as iron salts. Various kinds of magnetic nanoparticles have been fabricated employing this method, including iron oxide $\left(\mathrm{Fe}_{3} \mathrm{O}_{4}\right.$, $\mathrm{Fe}_{2} \mathrm{O}_{3}$ ), cobalt oxide, and nickel oxide nanoparticles (Bedja et al., 1994; Pannaparayil and Komarneni, 1989).

Since colloidal suspension of the nanoparticles in water is stable, the hydrolytic co-precipitation procedure is widely used in industry to produce nanoparticles (Reimer and Balzer, 2003). However, a disadvantage of this method is that it typically uncontrollably generates nanoparticles which have inhomogeneous sizes and shapes, and poor levels of crystallinity. These inhomogeneities hamper the use of the nanoparticles for rigorous measurements and investigations of nanoscale magnetism, as well as optimized applications. Consequently, most magnetic nanoparticles used in scientific studies are made employing the non-hydrolytic thermal decomposition method (Hyeon, 2003; Jun et al., 2007; Murray et al., 2001; Sun et al., 2004). In this technique, a solution of a metallic precursor in a water-free organic solvent containing appropriate surfactants is heated to cause thermal decomposition of the components and form the crystalline nanoparticle. This thermal decomposition process is easily controlled by adjusting the temperature, time, and type of precursor and surfactant. As a result, it can be employed to produce finely tuned materials in terms of size, shape, and composition (Hyeon, 2003; Jana et al., 2004; Jun et al., 2007; Murray et al., 2001; Sun et al., 2004). Various magnetic nanoparticles that have been made utilizing this method include metal ferrites, transition metal oxides, metals, and alloys (Murray et al., 2001; Sun, 2006; Sun et al., 2004). It is noteworthy that an anaerobic reaction environment is used in this method and, therefore, it can be employed to generate metallic or metal alloy nanoparticles that undergo oxidation readily in aqueous solution (Jana et al., 2004; Sun, 2006). Another advantage of the thermal decomposition method is that it can be used to produce nanoparticles with single crystallinity, an important feature governing magnetic properties. Magnetic properties are highly sensitive to crystalline defects, which cause formation of a magnetic grain boundary that leads to less than optimal magnetism.

In order to take full advantage of the non-hydrolytic thermal decomposition method, issues associated with the low aqueous solubilities and stabilities of the nanoparticles must be addressed. Since the nanoparticles are produced using this method as suspensions in organic solvents, they cannot be readily dispersed in aqueous solutions. Surface modification strategies, needed to change nanoparticle surfaces from hydrophobic to hydrophilic, have been developed to solve this problem (Latham and Williams, 2008; Tong et al., 2010; Zhang et al., 2002). Various substances ranging from small molecules to polymers and biomolecules possessing amphiphilic properties have been used as surface modifiers. These modifiers have ends that are designed to bind to the hydrophobic surfaces of the nanoparticle and other ends that have hydrophilic character to promote solubility in water. A well known example of substances of this type is meso-2,3-dimercaptossucinic acid (DMSA), which was developed by Lee et al. (2006) for surface modification of $\mathrm{Fe}_{3} \mathrm{O}_{4}$ nanoparticles. DMSA is dicarboxylic acids containing two thiol groups. One carboxylic acid group in this substance binds to the surface of the nanoparticle by serving as a Fe ion ligand and the other carboxylic acid moiety in its carboxylate ion form provides hydrophilic character. The thiol groups in DMSA serve as reactive centers that enable conjugation with other biomolecules or cross-linking with neighboring DMSAs on the nanoparticle surface. DMSA coated iron oxide nanoparticles are fairly stable in the colloidal state without undergoing aggregation in up to $200 \mathrm{mM} \mathrm{NaCl}$ solution in a $\mathrm{pH}$ range of $6-10$ (Lee et al., 2006).

Other surface modifiers commonly being used for this purpose are biologically safe and degradable polymers (Latham and Williams, 2008). Polyethyleneglycol (PEG) is typically employed owing to its anti-biofouling characteristics (Choi et al., 2010; Hong et al., 2005; Latham and Williams, 2006; Peng et al., 2006). PEG is highly soluble in aqueous solution and electrostatically neutral, properties which prevent self aggregation and electrostatic adsorption of plasma proteins in blood (Peng et al., 2006). PEG coated nanoparticles are reported to have high solubilities as well as colloidal stabilities in solutions of a wide range of salt concentrations and $\mathrm{pHs}$. However, PEG itself does not possess groups that enable binding to the surface of nanoparticles. As a result, appropriate modification of this substance is needed to introduce chemically active functional groups. Hydrophobic lipids or catechol moieties have been introduced at the one end of PEG for this purpose (Hong et al., 2005), as exemplified by the study of Choi et al. (2010a) in which PEGmodified lipids such as PEG-phosphatidylethanolamine (PEG$P E)$ and PEG-phosphatidylcholine (PEG-PC) were employed to produce magnetic nanoparticle $(\mathrm{MnO})$ encapsulated in micelles (Choi et al., 2010a). These micelles, comprised of PEGlipids, have a hydrophobic interior in which the hydrophobic nanoparticles reside without having direct contact with the outer aqueous environment. However, because the as formed structures are generally sensitive to changes in temperature and viscosity, micelles are transformed to more rigid structures by implementation of a secondary step involving cross-linking.

Another method for applying a PEG coating was developed by Peng et al. (2006). In this technique, a catechol group serving as a chelating ligand for metal atoms on the surfaces of magnetic nanoparticles is incorporated at one end of PEG. Owing to its high affinity for the metal atom, catechol conjugated PEG can be used to create a high density coating on the nanoparticle surface.

In addition, an inorganic surface coating method has been developed recently (Choi et al., 2010b; Yi et al., 2006). In this process, a silica precursor such as tetraethyl orthosilicate (TEOS) undergoes readily polymerization in the presence of base or acid catalysts through a sol-gel reaction mechanism. Typically, micelle forming surfactants are included in the polymerization media to control the size of the micelles, which in turn determines the thickness of silica coating (typically 5-50 nm) (Choi et al., 2010b) and the total nanoparticle size (Yi et al., 2006).

\section{MAGNETIC NANOPARTICLES AS MULTI-MODAL PLATFORMS}

The magnetic nanoparticles described above have found wide 


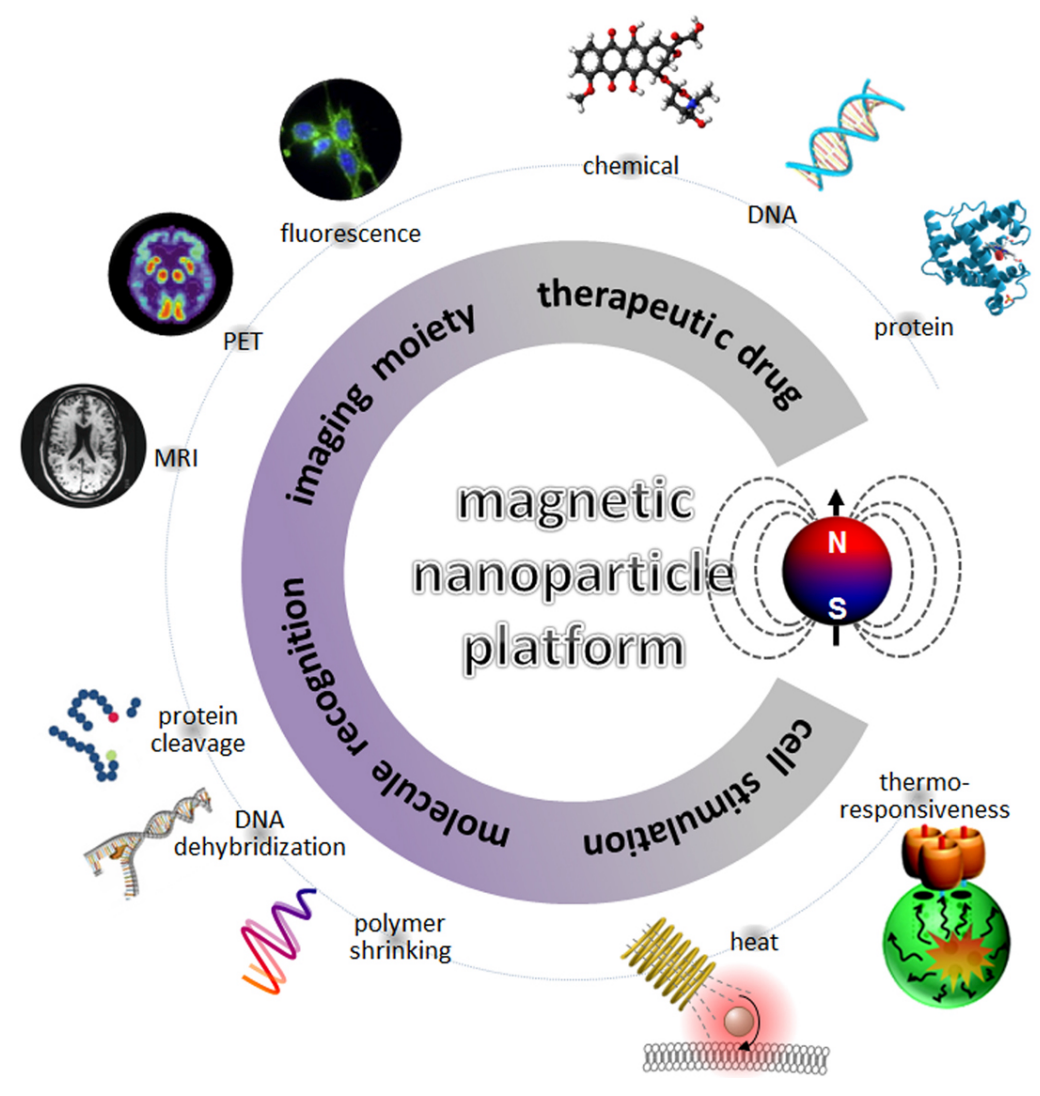

Fig. 1. Magnetic nanoparticles as platform materials for the integration of various modalities. Various imaging modalities and therapeutic drugs can be ligated onto magnetic nanoparticle. The linker molecule can also contain functionalities for the molecular recognition. Magnetic nanoparticle acts as stimuli, which trigger the activation of cell signaling processes inside cells. applications in medical areas, especially as contrast agents for magnetic resonance imaging (MRI), and magnetic drug delivery, biomolecule separation and magnetic hyperthermia treatment (Crick and Hughes, 1950; Glogauer et al., 1995; Valberg and Feldman, 1987; Wang et al., 1993). More recently, attempts have been made to combine each of these capabilities into one system, in which magnetic nanoparticles serve as multi-mode platforms for integrated applications. Multi-mode systems possess benefits of each modality operating in a synergistic manner. Various chemical moieties on the surfaces of nanoparticles can serve as conjugating sites where other imaging modalities or functional biomolecules can be ligated. For example, in order to enhance MRI contrast effects of magnetic nanoparticle, other imaging active moieties such as those containing fluorophores and/or radioisotopes can be added (Cheon and Lee, 2008). The additional ligating molecules can also be functionalized so that they sense the presence of specific biomolecules or environmental factors (Ghadiali and Stevens, 2008; Liu et al., 2009; Perez et al., 2002a). In addition, the magnetic nanoparticles can be employed as platforms to which a variety of therapeutically active chemical or biological agents can be conjugated to enhance the selectivity of hyperthermically active nanoparticles (Fig. 1). In the following sections, nanoparticle multimodality will be described with emphasis being given to the advantages these systems have in imaging and therapeutic applications.

\section{Multi-modal imaging}

The traditional multi-modal approach to magnetic nanoparticle based imaging combines MRI contrast effects with fluorescence emission (Lee et al., 2006; Ntziachristos, 2000). Although
MRI is well suited for non-invasive in vivo tomography, it lacks the resolution and sensitivity when applied to cellular systems. The typical resolution of clinical 1.5-3 T MRI is in the millimeter per volumetric pixel (voxel) range, which roughly comprises millions of cells. In most cases, a biopsy of the MRI identified tissue is needs to be performed to obtain histological information about the lesion and confirm the diagnosis. In this sense, the use of magnetic nanoparticles conjugated with fluorescence dyes is advantageous because it provides MRI imaging and fluorescence based histology simultaneously. In Figure 2A shows a representative MRI-fluorescence system comprised of an iron oxide nanoparticle ligated with the fluorescence dye, fluorescein-5-isothiocyanate (FITC) (Rodriguez et al., 2006). When injected intravenously into a mouse, in which prostate cancer xenografts have been implanted, the multi-modal nanoparticles accumulate in the implanted tumor region as a consequence of an enhanced permeability and retention (EPR) effect. The dark contrast seen in the T2 MRI clearly demonstrates the accumulation of nanoparticles in this region (Fig. 2B). In images of histological sections created by using fluorescence microscopy, the nanoparticles are clearly visible as green fluorescence areas embedded in the tumor tissue, which confirms the diagnosis of the cancerous nature of this region. Even though these results arose from a preliminary proof-of-concept study, they show that the dual-mode strategy can be applicable in a clinical environment.

One remaining issue, which needs to be technically resolved in this system, concerns fluorescence energy transfer between the dye and magnetic nanoparticles. Due to their huge light absorption cross-section, the magnetic nanoparticles quench 
A

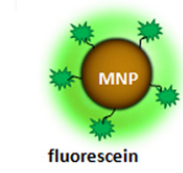

B
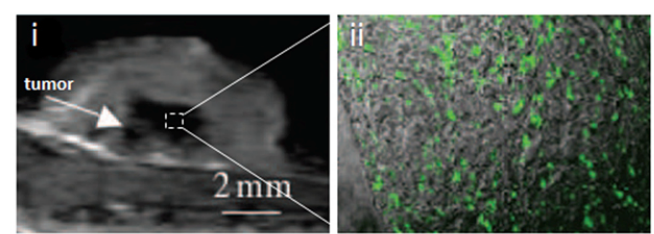

C

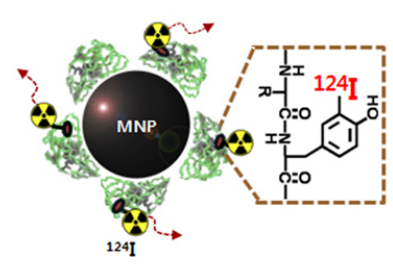

D

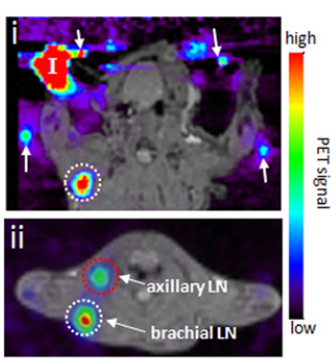

E

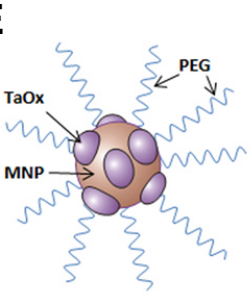

G

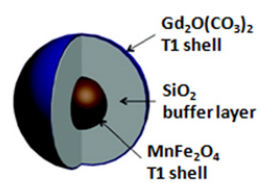

$\mathbf{F}$
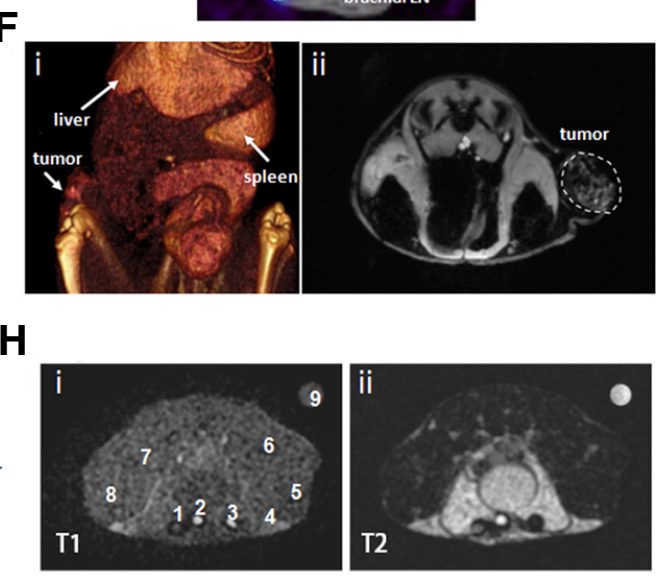

I

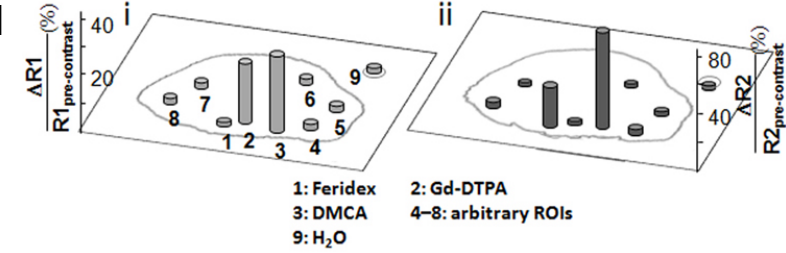

Fig. 2. Various multi-mode imaging systems based on magnetic nanoparticles. (A) MRI-fluorescence nanoparticle and (B) its MRI tumor targeted image and a magnified fluorescence histology image of the tumor region (Rodriguez et al., 2006). (C) MRI-PET dual-mode nanoparticle and (D) overlaid MRI-PET images in i) coronal and ii) axial view (Choi et al., 2008). (E) MRI-CT dual-mode nano-particle and (F) its 3D constructed CT (i) and MRI (ii) images of tumor in mouse (Lee et al., 2012). (G) T1-T2 dual-mode MRI nanoparticle and $(\mathrm{H})$ its i) T1 and ii) T2 image of mouse bearing phantom contrast agents in the abdomen region. (I) Measured signal of i) T1 and ii) T2 from images in (H) (Choi et al., 2010b). the excited state of the dye by energy transfer. This phenomenon results in a significant decrease of the fluorescence signal. However, the efficiency of the energy transfer process is highly dependent on the distance between the nanoparticles and dyes, decreasing as the distance increases. Consequently, energy transfer can be minimized by distancing the two modalities by more than ca. $10 \mathrm{~nm}$. Several strategies, employed to accomplish this goal, rely on the introduction of very long linker molecules between the dye and magnetic centers. Silica is especially useful in this regard owing to its rigid and transparent nature (Ow et al., 2005). Silica serves as a controllable thickness shell around nanoparticle which can position fluorescent dyes either on the inside or outside. Because fluorescent dyes reside at a remote and fixed position in the silica embedded magnetic nanoparticles, energy transfer or continuous blinking of fluorescence does not take place (Ow et al., 2005).

Another promising multi-modal system utilizes a combination of MRI and positron emission tomography (PET) (Choi et al., 2008b; Judenhofer et al., 2008). While MRI is designed for highly resolved tomographic imaging of the body with a spatial resolution down to hundreds microns, the PET imaging modality based on gamma rays emitted from decaying radioisotopes has extreme sensitivity extending down to the picogram $\left(10^{-12}\right.$ grams) per milliliter level (Massoud and Gambhir, 2003). As a result of their independent advantageous features, MRI and PET can be combined to create a dual modality system that displays nearly ideal in vivo imaging properties associated with superior spatial resolution and sensitivity.

PET-MRI imaging has been performed clinically by using sequential injections of PET and MRI contrast agents (Henze et al., 2001). However, this technique is limited by the different pharmacokinetic properties of the two contrast agents, often giving rise to unmatched regions in both images. In order to overcome this problem, Choi et al. (2008b) developed multimodal contrast agent for PET-MRI imaging, in which magnetic nanoparticles $\left(\mathrm{MnFe}_{2} \mathrm{O}_{4}\right)$ are conjugated with ${ }^{124} \mathrm{I}$, a radioisotope used for PET imaging. To introduce ${ }^{124} \mathrm{I}$, the $\mathrm{MnFe}_{2} \mathrm{O}_{4}$ nanoparticles are first coated with a biologically irrelevant protein (e.g., serum albumin) and then mixed with ${ }^{124} \mathrm{ICl}$ to promote the conjugation reaction. In this case, ${ }^{124} \mathrm{I}$ is introduced only at 
A

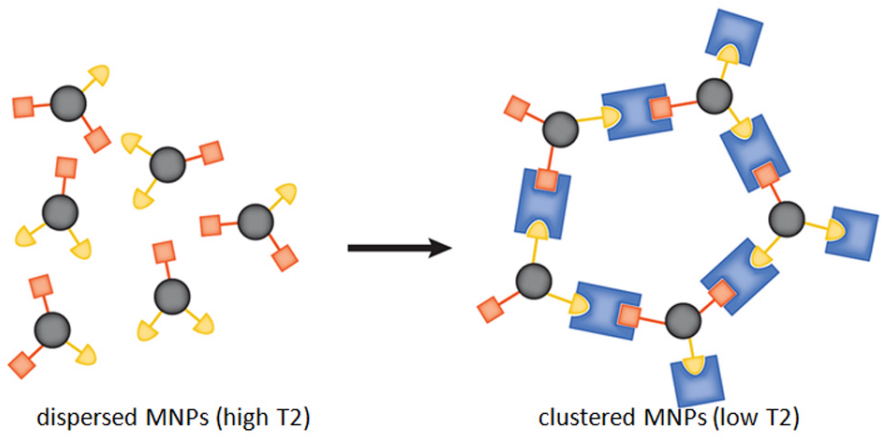

B

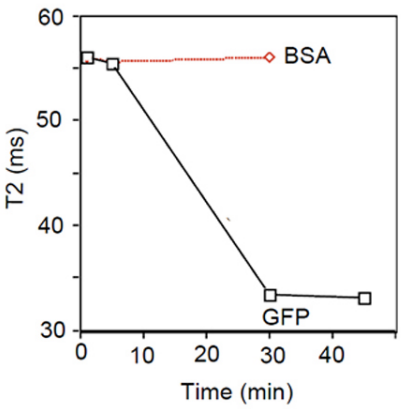

C

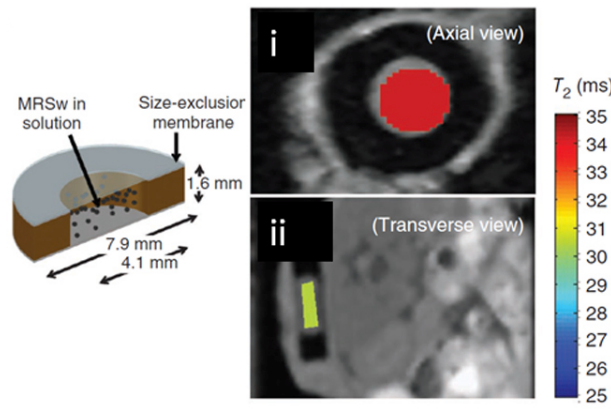

Fig. 3. Molecular recognition using a magnetic relaxation switch. (A) Scheme for a magnetic relaxation switch. Dispersed magnetic nanoparticle in high T2 can be assembled into aggregated structures in the presence of target molecules, decreasing the T2 relaxation time (Apple, 2011). (B) A graph of the detection of the target protein, GFP. The magnetic nanoparticles are mixed with the target GFP protein, nanoparticles become aggregated with a significant decrease of T2 within $30 \mathrm{~min}$ (Perez et al., 2002b). (C) Implantable device, which can detect cancer marker in vivo, and the mouse MRI of implanted device (axial view and transverse view) (Ling et al., 2011). the ortho-positions of the aromatic rings of tyrosine residues in the protein (Fig. 2C). The resulting PET-MRI contrast agent was observed to be effective for in vivo imaging of lymph nodes. Although detection of sentinel lymph nodes is important for diagnosing metastasis of tumors, it has not often performed using MRI because of the small sizes of the lymph nodes. PET provides the high sensitivity required for this purpose but it generally lacks the ability to provide information about the anatomy of tissues. However, the combined PET-MRI images system provides images that are helpful in arriving at a diagnosis of sentinel lymph nodes. As seen by inspection of the images in Fig. 2D, the combined PET and MRI agent has the sensitivity and resolution required to show that nanoparticles injected into the front paw of mouse reach the sentinel lymph node (see the axial and coronal images of combined PET-MRI). This is an excellent example of an application of PET-MRI multi-mode contrast agents, which should become more widespread because of the recent advances made in the development of instruments which can simultaneously record PET and MRI images (Choi et al., 2008b).

Applications of MRI imaging can be extended by combining it with the computed tomography (CT) imaging technique. CT and MRI are currently used in clinical applications as alternative imaging modalities that provide similar results owing to their intrinsic high resolution and non-invasive tomography characteristics. However, these techniques display subtle differences reflected in the fact that CT has a greater capability in computational reconstruction of high resolution $3 \mathrm{D}$ images but gives weaker contrast in soft-tissue analysis. In comparison, T2 MRI is a superior method for obtaining high sensitivity soft-tissue contrast images but it suffers from false-positive artifact signals. Consequently, combining these two modalities in one system would be beneficial for the accurate diagnosis of cancers. Recently, a dual modality MRI-CT contrast agent has been developed that is comprised of iron oxide nanoparticles containing a tantalum oxide (TaOx) shell (Fig. 2E) (Lee et al., 2010). Tantalum is an electron rich transition metal which readily absorbs $X$ rays and provides higher contrast CT images (Bonitatibus et al., 2010; Oh et al., 2011). To apply this core-shell structured nanoparticle to in vivo analysis, the surface was modified with silica and PEG to ensure biological stability and colloidal dispersity. When injected intravenously into a rat implanted with mammary adenocarcinoma tumor, this nanoparticle enables clear imaging of the tumor region in both CT and MRI modes (Fig. 2F). In this preliminary proof-of-concept study, a very high dosage [840 $\mathrm{mg} / \mathrm{kg}$ (drug/body)] of the nanoparticles was required, a limitation that needs to be resolved before the technique can become generally applicable for MRI-CT dual-mode imaging (Lee et al., 2012).

Another possible combination of imaging modalities fuses $\mathrm{T} 1$ and T2 MRI into one system. T1 and T2 are different imaging modes in MRI, in which T1 measures the proton longitudinal spin relaxation time (spin-lattice relaxation) and T2 measures its transverse spin relaxation time (spin-spin relaxation). Faster relaxation times in both of these modes result in higher contrasts, corresponding to whiter signals for T1 and darker signals in T2. Different contrast agents including paramagnetic molecular substances (eg., $\mathrm{Gd}^{2+}$ or $\mathrm{Mn}^{2+}$ chelate compounds) and superparamagnetic nanoparticle agents (eg., iron oxide-based nanoparticles) have been employed to enhance the respective T1 and T2 relaxation times (Arbab et al., 2006; Jun et al., 2008b; Lauffer, 1987; Na et al., 2009). However, independent T1 or T2 based imaging still suffers from false-positive signals ubiquitously observed in tissues. As a result, in most cases a comparison of T1 and T2 images is required in order to increase diagnose accuracy. In typical imaging processes, the T1 and T2 images are recorded following sequential injections of T1 and T2 contrast agents (Kubaska et al., 2001; Maccioni et al., 2006). However, the different pharmacokinetics of these agents makes the entire imaging procedure complicated and 
A

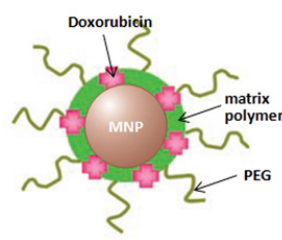

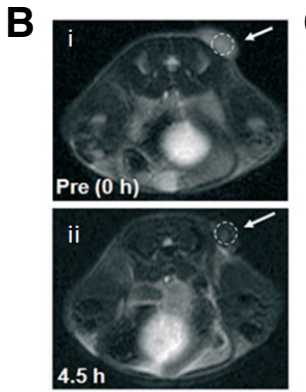

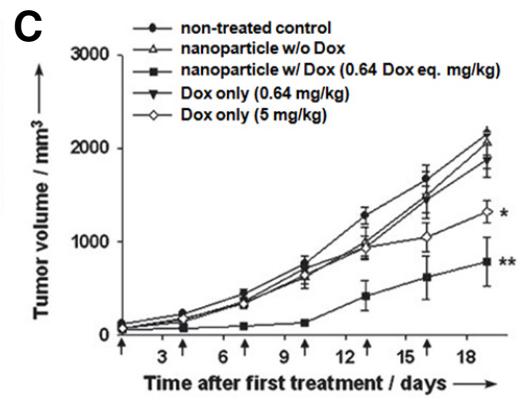

D

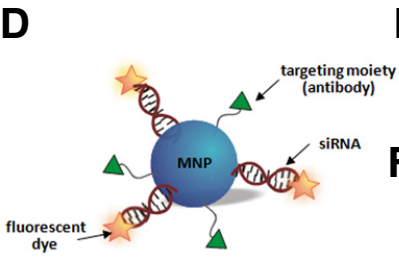

E

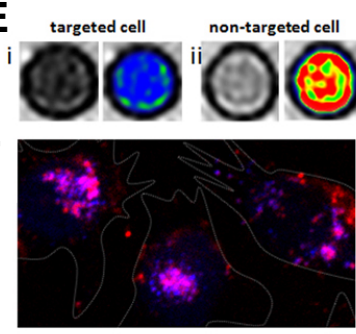

H

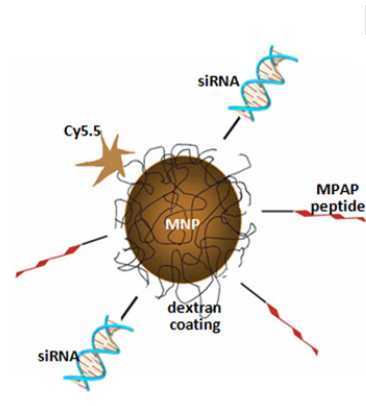

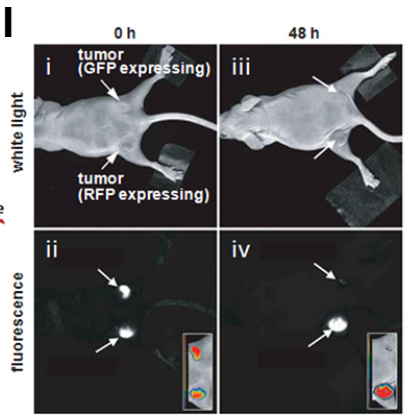

G

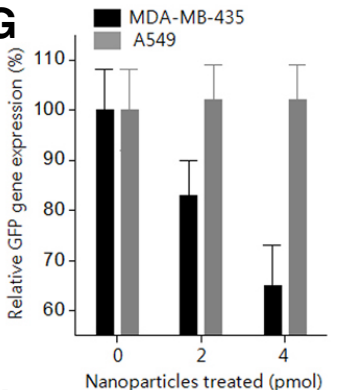

$J$

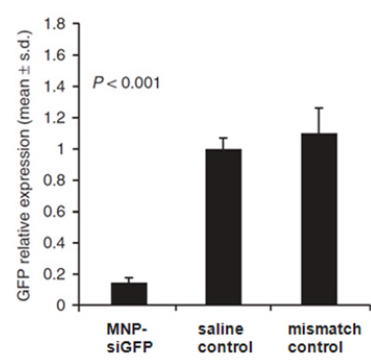

Fig. 4. Multi-mode systems for simultaneous imaging and drug delivery. (A) Magnetic nanoparticle conjugated with doxorubicin for achieving MRI and drug release at the same time. (B) $\mathrm{MRI}$ images of tumor (white arrow) bearing mouse i) before and ii) after injection of nanoparticles, and (C) a graphs on tumor volume vs. time after nanoparticle treatments. Doxorubicin conjugated nanoparticles lowered the growth rate of tumor (Yu et al., 2008). (D) Magnetic nano- particle designed to deliver siRNA and multimode imaging. $(\mathrm{E})$ In vitro $\mathrm{MRI}$ of nanoparticle treated cells, i) targeted cancer cells (MDA-MB-435) and ii) nontargeted cells (A549). (F) Immunofluorescence confocal image of MDA-MB-435 treated with nanoparticles (red: nanoparticle, blue: endosome, pink: nanoparticle + endosome). (G) A graph of GFP expression levels along with the amounts of nanoparticle treatments (Lee et al., 2009). (H) Magnetic nanoparticles designed to deliver siRNA to tumor in vivo. (I) White light and fluorescence pictures of mouse i, ii) before and iii, iv) after injection of nanoparticles. Nanoparticles successfully targets the tumor and suppresses the expression of GFP in tumor. (J) A graph of GFP expression levels when the nanoparticle and other negative controls are treated to tumor in mouse (Medarova et al., 2007).

dual injections of contrast agents can be a burden to a patient.

Several attempts have been made to develop simultaneous T1 and T2 imaging methods that utilize single contrast agents, in which for example the agents are ligated. This approach was eventually useless as a consequence of magnetic spin interactions occurring between the T1 and T2 materials. T2 agents (magnetic nanoparticle) have their own magnetic gradient fields which perturb the spin relaxation behavior of neighboring $\mathrm{T} 1$ agents (paramagnetic chelate molecule) (Yi et al., 2006). This cross interaction results in the loss of $\mathrm{T} 1$ contrast effects which depends on the distance between the T1 and T2 agents.

This problem has been overcome in studies that led to the development of dual-mode nanoparticle contrast agents (DMCA) (Fig. 2G) (Choi et al., 2010b). The DMCA nanoparticle has a core-shell structure comprised of an iron oxide nanoparticle core, which serves as the T2 contrast agent, and a shell containing a Gd-based T1 agent. This dual-mode nanoparticle also possesses a ca. $10 \mathrm{~nm}$ silica layer between the T1 and T2 agents which acts as a buffer to minimize the magnetic spin interactions between the two agents. The results of experiments showed that DMCA displays comparable or even higher $\mathrm{T} 1$ and $\mathrm{T} 2$ contrast effects than the related single mode $\mathrm{T} 1$ and T2 contrast agents, Gd-DTPA and iron oxide nanoparticle, respectively. A significant advantage of the DMCA system arises from its AND logic image-filtering capability (Choi et al., 2010b). AND logic process is a Boolean logic process, which yields 'true' signals only when both input signals are 'true'. Following DMCA introduction into the body, the recorded T1 and T2 images are subjected to AND processing to remove all falsepositive signals and produce the true signal. In Fig. $2 \mathrm{H}$ are shown the T1 and T2 MRI of DMCA, T1 agent (Gd-DTPA), and T2 agent (Feridex) phantoms implanted into abdomen region of mice. The signal intensities of regions of interest (1-9) in these images (including the region of DMCA, Gd-DTPA, and Feridex implants) were measured. Signals from all irrelevant areas (number 4-9) were ignored because of their low intensities. Signals from Gd-DTPA and Feridex are sufficiently high in only the individual T1 and T2 MRI images, respectively, and as a result they are canceled in the AND process. Because only the signal from DMCA is intense in both the T1 and T2 modes and, as a result, it survives the AND process (Fig. 2l). Although preliminary, these results suggest that an opportunity exists for the wide use of T1-T2 contrast agents coupled with AND logic processing to produce accurate images for diagnoses.

\section{Imaging based on molecular recognition and sensing}

Recently, investigations of magnetic nanoparticle imaging systems have entered a new phase in which techniques involving 


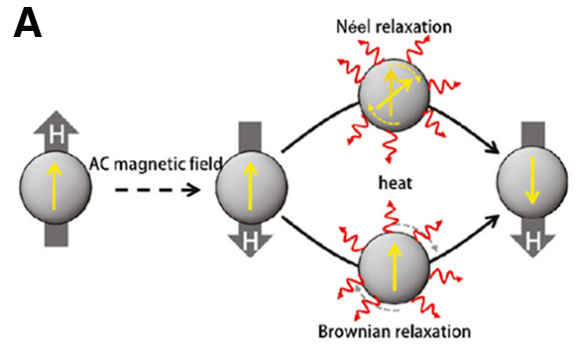

B

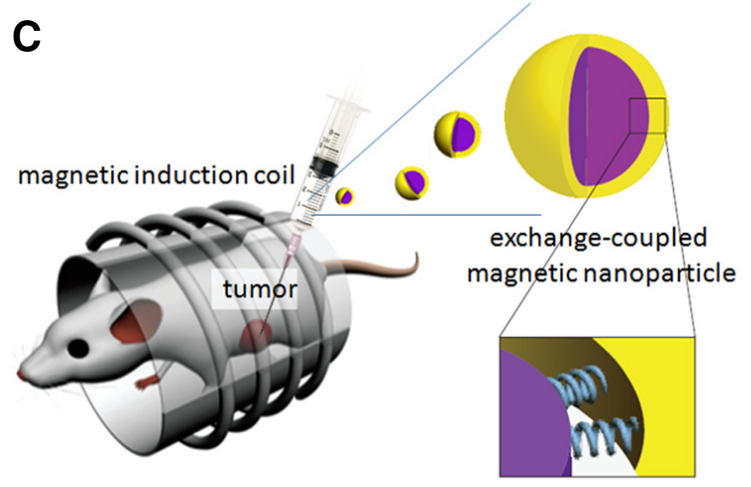

E

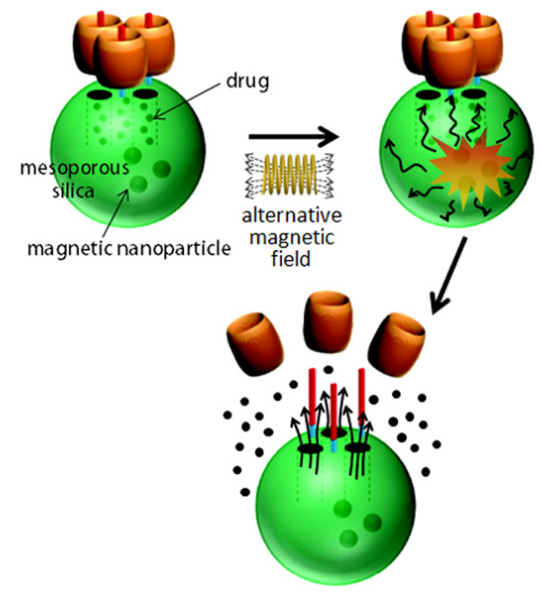

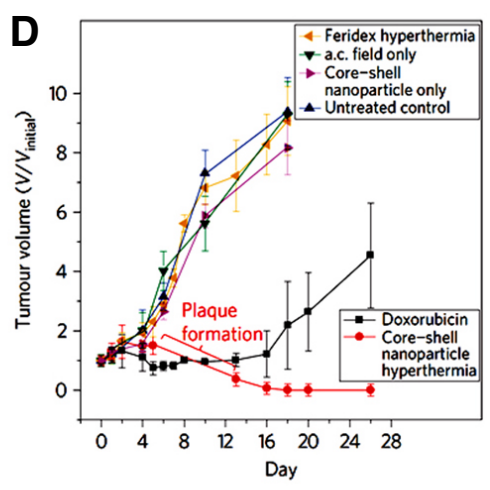
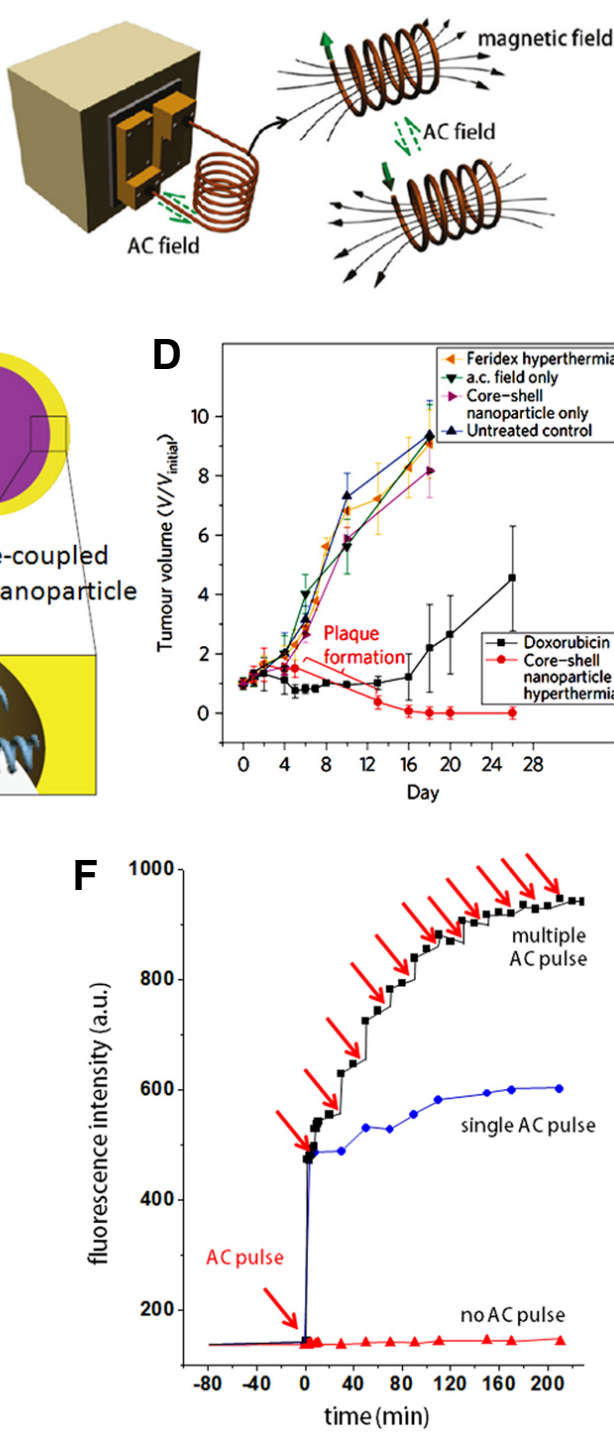

Fig. 5. (A) Basic principle of magnetic heat induction with Néel and Brownian relaxation. (B) The coil set-up for generating alternative magnetic field (AMF) with 100$1,000 \mathrm{kHz}$ (Yoo et al., 2011). (C) Magnetic hyperthermia therapy of a tumor in mouse. Exchange coupled magnetic nanoparticle exhibiting superior heat induction upon intratumoral injecteion. (D) Graph of temporal changes of tumor mass in mouse, treated with magnetic hyperthermia and other control groups (Lee et al., 2011). (E) A scheme of a stimuli-responsive drug release system using magnetic nanoparticle. Magnetic nanoparticles are inside mesoporous nanoparticle with a chemical drug. The mesoporous channel is blocked with molecular caps. Upon the irradiation of AMF, the heat emitted from the magnetic nanoparticle increases the pressure inside nanoparticle, and leads to release of the chemical drug. (F) Temporal drug release profile measured with fluorescence intensity of the drug. Red arrows indicate the times AMF was applied (Thomas et al., 2010). molecular recognition and sensing are being developed (Lewin et al., 2000; Weissleder et al., 2000). Typically in MRI or other imaging methods, magnetic nanoparticles perform the single function of either reporting or transducing their exact location to the outside. It is now recognized that through conjugation, magnetic nanoparticles can actually perform the secondary function of reporting the presence and quantities of target biomolecules. Perez et al. (2002b) were the first to develop magnetic nanoparticles that have this sensory function, which they termed "magnetic relaxation switches". This detection system relies on the fact that different T2 magnetic relaxation times exist when magnetic nanoparticles are in isolated versus clustered states (Fig. 3A) (Apple, 2011). Because clustered magnetic nanoparticles behave like bigger particles, their magnetization values are elevated. For this reason, the clustered nanoparticles have enhanced relaxation times, which are observed as darker signals in MRI. Perez et al. (2002b) utilized this phenomenon in devising an active biomolecule conjugated nanoparticle, which can aggregate or be cleaved in the presence of specific sub- stances or enzymes. An example of this approach (Fig. 3B), is found in the use of magnetic nanoparticles conjugated with antiGFP antibody to detect the presence of green fluorescence protein (GFP) through a change in the T2 relaxation time from 56 to $33 \mathrm{~ms}$. Employment of this technique to detect an enzyme (e.g., caspase 3 ) or specific DNA at femto-molar levels was also demonstrated in this effort (Perez et al., 2002b). Moreover, because changes in T2 relaxation times can be measured precisely, this method can be applied in a quantitative manner. Recently, Ling et al., (2011) utilized this magnetic relaxation switch system for detection and temporal in vivo monitoring of cancer markers in a mouse model. In this work, a chamber, containing antibody conjugated magnetic nanoparticles and sealed with size exclusion membrane, was implanted in a mouse tumor (Fig. 3C). The cancer marker released from the mouse tumor are detected by the embedded nanoparticles that serve as a magnetic relaxation switch with $\mathrm{pg} / \mathrm{ml}$ precision level for up to $72 \mathrm{~h}$ (Ling et al., 2011).

Other studies of magnetic relaxation switches have focused 
on the development of systems to detect and quantify matrix metalloproteinase (MMP) and the amyloid-beta protein (Choi et al., 2008a; Harris et al., 2006). For detection of MMP in the vicinity of a cancerous tumor, magnetic nanoparticles were conjugated with a MMP-cleavable peptide (Harris et al., 2006). In the presence of MMP, the peptide is cleaved enabling clustering of the released magnetic nanoparticles. Because MMP is a key protein secreted from cancerous tumors, this magnetic relaxation switch has the potential of being used to diagnose tumors. In a similar fashion, amyloid-beta protein agglomeration can be detected quantitatively by using appropriately conjugated magnetic nanoparticles (Choi et al., 2008a). As a consequence of the fact that amyloid-beta protein causes Alzheimer's disease in a manner that is dependent on its degree of agglomeration, the magnetic relaxation switch developed in this effort can be utilized in the diagnosis or prognosis of Alzheimer's disease.

\section{Imaging combined with drug delivery}

As described above, magnetic nanoparticles can be used directly as drugs or as platforms for drug delivery when conjugated to various chemical or biological substances. In this sense, along with their MRI contrast enhancing capabilities, magnetic nanoparticles can be employed as multi-mode platforms for simultaneous imaging and drug delivery (Cheon and Lee, 2008; Yoo et al., 2011). The term "theranosis" has been used to describe this type of combined therapy and diagnosis (Yoo et al., 2011).

One example of this approach employs magnetic nanoparticles that are conjugated with the common antitumor agent doxorubicin. In this effort, Yu et al. (2008) synthesized iron oxide nanoparticles whose surfaces are coated with a polymer matrix comprised of PEG to provide an anti-biofouling capability that enables the nanoparticles to circulate inside the body without severe flocculation. Next, doxorubicin was conjugated with the coated nanoparticles to provide a therapeutic capability (Fig. 4A). Following their injection into a mouse model, containing human tumor xenographs, the doxorubicin modified magnetic nanoparticles accumulate in the tumor mass by the EPR effect. The nanoparticles were then successfully imaged by using T2 $\mathrm{MRI}$, in which the contrast associated with the tumor changes from light to dark at $4.5 \mathrm{~h}$ post injection (Fig. 4B). In addition, the size of the tumor mass was monitored for 20 days period with nanoparticle injections occurring every 3 days. The results show that the growth rate of the tumor mass is decreased in the nanoparticle injected mice compared to that of a control group (Fig. 4C) (Yu et al., 2008). However, repetitive treatments with the doxorubicin conjugated nanoparticles did not lead to complete disappearance of the tumor mass, which is in part a consequence of the limited loading of doxorubicin and low targeting efficiency in vivo.

As another example of the theranostic approach involves conjugation of a therapeutic gene to nanoparticles. Recently, small interfering RNAs (siRNAs) have received great attention owing to their therapeutic potential for inhibiting the expression of important proteins in cancer cells. Because safe delivery of large quantities of siRNAs to target cells is an important issue to address in this field, methods based on magnetic nanoparticles have been explored. By using this type of an approach, the delivery process can be monitored by using MRI. We have provided a demonstrative example of this strategy, in which iron oxide magnetic nanoparticles are attached with a GFP expression specific siRNA, antibody and fluorescent dye (Fig. 4D) (Lee et al., 2009). The antibody attached to the nanoparticles provides selective targeting ability to only cancer cells, and the magnetic nanoparticle and fluorescent dye provide the multimode imaging capability associated with MRI and immunofluorescence (Figs. 4E and 4F). Once attached to surfaces of cancer cells, the nanoparticles are endocytosed by an enzymatic cleavage reactions leading to detachment of siRNAs. In this effort, the effect of the released siRNAs on cancer cells was demonstrated by observing that GFP expression gradually decreases in a manner that is proportional to the amount of nanoparticles used (Fig. 4G) (Lee et al., 2009).

Medarova et al. (2007) provided an example of this technique for simultaneous in vivo imaging and siRNA delivery, which utilized iron oxide magnetic nanoparticles linked to a green fluorescence protein (GFP) suppressive siRNA and the MPAP, a membrane translocation peptide that aids entry of the nanoparticle into the cell (Fig. $4 \mathrm{H})$. In addition, the nanoparticles were coated with dextran to ensure their colloidal stability as well as linked to the dye Cy5.5 to provide fluorescence visibility. The results of in vivo MRI and fluorescence imaging (Figs. 4I and $4 \mathrm{~J}$ ) studies indicate that the nanoparticles do indeed target the tumor and inhibit the expression of GFP.

In addition to these studies, several other interesting investigations have been carried out to demonstrate the theranostic capabilities of magnetic nanoparticles. For example, Lee et al. (2010) demonstrated that dye-doped silica nanoparticles coated with magnetic nanoparticles and doxorubicin can be employed to image and therapeutically affect tumors. Although most investigations of magnetic nanoparticle are at a very early stage and complete elimination of tumors has not yet been achieved, the results accumulated thus far show that the theranostic related multi-modal capabilities of magnetic nano-particles hold great promise in cancer imaging/chemotherapy.

\section{MAGNETIC NANOPARTICLES AS THERAPEUTIC PLATFORMS}

As the magnetic properties of nanoparticles are developing in terms of magnetization value, coercivity, and anisotropy, the nanoparticle's heat induction properties have been drawn significant interest in these days for magnetic hyperthermia therapy. Magnetic hyperthermia utilizes heat emitted from magnetic nanoparticles, promoted by application of an external high frequency alternating magnetic field (AMF). In this process, the internal magnetic spins of nanoparticles relax in phase and continuously upon application of an AMF of $100-1000 \mathrm{kHz}$ as a consequence of a combination of Néel and Brown processes. While the spins relax, energy associated with transitions between the up and down spin states is emitted as heat (Figs. 4A and $4 \mathrm{~B}$ ). Because magnetic fields are not significantly attenuated upon transmission through biological tissues, they can be utilized for hyperthermia therapy of deeply buried tumors (Derfus et al., 2007). Moreover, cancer cells are usually more sensitive to heat than benign cells, with the typical temperature initiating apoptosis of cancer cells being ca. $42-45^{\circ} \mathrm{C}$ (Harmon et al., 1990; O'Neill et al., 1998). Heat induction from the magnetic nanoparticles, typically expressed as specific loss power (SLP), is proportional to the size, magnetization value, and magnetic anisotropy of nanoparticles (Lee et al., 2011; Noh et al., 2012), as well as their concentrations in tissues, and intensity and frequency of AMF. Importantly, the heat produced in this manner is sufficient to provide the temperature range for cancer cell apoptosis (Hergt et al., 2006).

Iron oxide nanoparticles $\left(\gamma-\mathrm{Fe}_{2} \mathrm{O}_{3}\right)$ have been generally used for in vivo and clinical magnetic hyperthermia therapy carried 
out during the last 10 years (Wust et al., 2002). This form of therapy is clinically approved in Europe for treatments of glioblastoma (Maier-Hauff et al., 2011; http://www.magforce.de/en). However, limitations which prohibit general application of this therapy are associated with the low treatment efficacy even if high concentrations of nanoparticles are employed (Hergt et al., 1998). As a result, lowering dose levels without compensating therapeutic effects is one of the goals of continuing magnetic hyperthermia research. Various magnetic nanoparticles have been developed with this goal in mind. In one effort, Lee et al. (2011) have developed a core-shell type magnetic nanoparticle, which exhibits exchange-coupled magnetism (Fig. 5C). The developed core-shell nanoparticle has tunable magnetic anisotropy, which leads to SLP values over $3000 \mathrm{~W} \mathrm{~g}^{-1}$ that are nearly 30 times higher than those of conventional magnetic nanoparticles. Observations made in this investigation show that when these nanoparticles are injected into a $100 \mathrm{~mm}^{3}$ size tumor implanted into a mouse, the tumor is eliminated completely after 15 days, a feat that is impossible to accomplish using conventional chemotherapies (Fig. 5D) (Lee et al., 2011).

Besides employing them to directly heat cancer cells, nanoparticles can be used in a more elegant way. A representative example of this novel approach was provided by Thomas et al. (2010), in which magnetic nanoparticles were embedded inside mesoporous silica nanoparticles. The silica nanoparticles contained the anti-cancer drug, doxorubicin, within microchannels in the mesoporous structure that were then capped by using a readily degradable molecular structure. The basic concept utilized to design this hybrid system is embodied in the following steps: (1) AMF heats the magnetic nanoparticles, (2) the heat increase causes the inner pressure to increase, and (3) when inner pressure reaches certain threshold level, the molecular caps disengage to release the drug molecules (Fig. 5E). It is worth noting that the important advantage of this system is that drug release can be controlled precisely by the AMF, which serves as a trigger, to promote drug release in spatio-temporally controlled manner. This ability contrasts with those of traditional drug delivery systems, which are limited by their passive kinetics of drug release. Because of this feature, drugs inside traditional delivery vehicles are continuously and uncontrollably released (Giri et al., 2005; Lai et al., 2003). However, the new hybrid system does not suffer from this drawback but, instead, quantitative amounts of the drug are released in an on-demand manner. In Fig. $5 \mathrm{~F}$ is show this controlled release behavior in which ca. $40 \%$ of the drug is released upon the first AMF stimulation and the rest being released from the nanoparticle in a staircase-like fashion upon sequential AMF stimulations. Importantly, leakage of the drug from the nanoparticle does not take place in this system and the release of the active drug effectively induces apoptosis and necrosis of cancer cells (Thomas et al., 2010). Similar strategies have been devised recently in the form of an AMF induced DNA releasing system and a double-effector magnetic nanoparticle, apoptotic hyperthermia system (Dobson, 2006). The latter double-effector system contains magnetic nanoparticles that are conjugated to a substance that generates a reactive oxygen species (ROS) (Yoo et al., 2012). When this nano-particle is transfected into cancer cells, the generated ROS act to sensitize cancer cells to be more susceptible to hyperthermia induced apoptosis.

\section{CONCLUSION}

Since the advent of methods to synthesize magnetic nanoparticles, a continuous effort has been given to their bio-medical applications (Cheon and Lee, 2008; Yoo et al., 2011). Owing to the development of nanotechnologies, various promising applications have been devised to the point at which now magnetic nanoparticles are among the most promising materials for nanomedicine. The MRI contrast enhancement, magnetic separation and drug delivery, and magnetic hyperthermia properties of magnetic nanoparticles have individually contributed to applications of this technology to nanomedicine. It is perhaps a natural outcome that these properties are now being combined with other biotechnological methods to create multi-modal nanoparticle based systems with enhanced synergistic functions. Here, we briefly discussed studies leading to the development of these multi-modal approaches to nanomedical imaging and therapeutics, which have demonstrated their great potential. Also, we have reviewed recent advances made in the new field of theranosis, which show that magnetic nanoparticles can serve as core materials in systems capable of being transformed from the laboratory bench to the clinical environment. For this purpose, additional investigations are needed to optimize and maximize the properties of the new nanoparticles. In addition, assessments of pharmacokinetics and pharmacodynamics are also crucial component of developing the viability of magnetic nanoparticle-based applications. Although some of the tasks that remain to be accomplished in this new area are labor and cost intensive and are difficult to perform by single academic laboratories, they are important when the therapeutic consequences of the results are taken into account.

\section{ACKNOWLEDGMENTS}

This research was supported by National Creative Research Initiative (2010-0018286), and World Class University (WCU) (R32-10217)

\section{REFERENCES}

Alivisatos, A.P. (1996). Perspectives on the physical chemistry of semiconductor nanocrystals. J. Phys. Chem. 100, 13226-3239.

Apple, F.S. (2011). Biomarkers in aggregate. Nat. Biotechnol. 29, 236-237.

Arbab, A.S., Liu, W., and Frank, J.A. (2006). Cellular magnetic resonance imaging: current status and future prospects. Expert Rev. Med. Devices 3, 427-439.

Bedja, I., Hotchandani, S., and Kamat, P.V. (1994). Preparation and photoelectrochemical characterization of thin SnO2 nanocrystalline semiconductor films and their sensitization with bis $\left(2,2^{\prime}\right.$ bipyridine)(2,2'-bipyridine-4,4'-dicarboxylic acid)ruthenium(ii) complex. J. Phys. Chem. 98, 4133-4140

Bigioni, TP., Lin, XM., Nguyen, TT., Corwin, El., Witten, TA., and Jaeger, H.M. (2006). Kinetically driven self assembly of highly ordered nanoparticle monolayers. Nat. Mater. 5, 265-270.

Bonitatibus, P.J.Jr., Torres, A.S., Goddard, G.D., FitzGerald, P.F and Kulkarni, A.M. (2010). Synthesis, characterization, and computed tomography imaging of a tantalum oxide nanoparticle imaging agent. Chem. Commun. 46, 8956-8958.

Cheon, J., and Lee, J.H. (2008). Synergistically integrated nanoparticles as multimodal probes for nanobiotechnology. Acc. Chem Res. 41, 1630-1640.

Choi, J.S., Choi, H.J., Jung, D.C., Lee, J.H., and Cheon, J. (2008a) Nanoparticle assisted magnetic resonance imaging of the early reversible stages of amyloid beta self-assembly. Chem. Commun. 2197-2199.

Choi, J.S., Park, J.C., Nah, H., Woo, S., Oh, J., Kim, K.M., Cheon G.J., Chang, Y., Yoo, J., and Cheon, J. (2008b). A hybrid nanoparticle probe for dual-modality positron emission tomography and magnetic resonance imaging. Angew. Chem. Int. Ed. Engl. 47, 6259-6262.

Choi, J.Y., Lee, S.H., Na, H.B., An, K., Hyeon, T., and Seo, T.S. (2010a). In vitro cytotoxicity screening of water-dispersible metal oxide nanoparticles in human cell lines. Bioprocess Biosyst. Eng. 33, 21-30. 
Choi, J.S., Lee, J.H., Shin, T.H., Song, H.T., Kim, E.Y., and Cheon, J. (2010b). Self-confirming "AND" logic nanoparticles for faultfree MRI. J. Am. Chem. Soc. 132, 11015-11017.

Crick, F.H.C., and Hughes, A.F.W. (1950). The physical properties of cytoplasm - a study by means of the magnetic particle method .1. experimental. Exp. Cell Res. 1, 37-80.

Cullity, B.D. (1972). Introduction to Magnetic Materials (AddisonWesley Publishing: Reading).

Dabbousi, B., RodriguezViejo, J., Mikulec, F., Heine, J., Mattoussi, H., Ober, R., Jensen, K., and Bawendi, M.G. (1997). (CdSe)ZnS core-shell quantum dots: synthesis and characterization of a size series of highly luminescent nanocrystallites. J. Phys. Chem. B 101, 9463-9475.

Derfus, A.M., von Maltzahn, G., Harris, T.J., Duza, T., Vecchio, K.S., Ruoslahti, E., and Bhatia, S.N. (2007). Remotely triggered release from magnetic nanoparticles. Adv. Mater. 19, 3932-3936

Dobson, J. (2006). Magnetic nanoparticles for drug delivery. Drug Dev. Res. 67, 55-60.

Ferrari, M. (2005). Cancer nanotechnology: opportunities and challenges. Nat. Rev. Cancer 5, 161-171.

Fortin, J.P., Wilhelm, C., Servais, J., Menager, C., Bacri, J.C., and Gazeau, F. (2007). Size-sorted anionic iron oxide nanomagnets as colloidal mediators for magnetic hyperthermia. J. Am. Chem. Soc. 129, 2628-2635.

Ghadiali, J.E., and Stevens, M.M. (2008). Enzyme-responsive nanoparticle systems. Adv. Mater. 20, 4359-4363.

Giri, S., Trewyn, B.G., Stellmaker, M.P., and Lin, V.S.-Y. (2005). Stimuli-responsive controlled-release delivery system based on mesoporous silica nanorods capped with magnetic nanoparticles. Angew. Chem. Int. Ed. Engl. 44, 5038-5044.

Glogauer, M., Ferrier, J.C., and McCulloch, A. (1995). Magneticfields applied to collagen-coated ferric-oxide beads induce stretchactivated $\mathrm{ca}^{2+}$ flux in fibroblasts. Am. J. Physiol. Cell Physiol. 269, 1093-1104.

Gupta, A.K., and Gupta, M. (2005). Synthesis and surface engineering of iron oxide nanoparticles for biomedical applications. Biomaterials 26, 3995-4021.

Harmon, B.V., Corder, A.M., Collins, R.J., Gob, G.C., Allen, J., Allan, D.J., and Kerr, J.F. (1990). Cell-death induced in a murine mastocytoma by 42-47-degrees-c heating in vitro - evidence that the form of death changes from apoptosis to necrosis above a critical heat load. Int. J. Radiat. Biol. 58, 845-858.

Harris, T.J., von Maltzahn, G., Derfus, A.M., Ruoslahti, E., and Bhatia, S.N. (2006). Proteolytic actuation of nanoparticle selfassembly. Angew. Chem. Int. Ed. Engl. 45, 3161-3165.

Henze, M., Schuhmacher, J., Hipp, P., Kowalski, J., Becker, D.W., Doll, J., Mäcke, H.R., Hofmann, M., Debus, J., and Haberkorn, U.J. (2001). PET imaging of somatostatin receptors using [(68)GA] DOTA-D-Phe(1)-Tyr(3)-octreotide: First results in patients with meningiomas. J. Nucl. Med. 42, 1053-1056.

Hergt, R., Andra, W., d'Ambly, C.G., Hilger, I., Kaiser, W.A., Richter, U., and Schmidt, H.G. (1998). Physical limits of hyperthermia using magnetite fine particles. IEEE Trans. Magn. 34, 3745-3754.

Hergt, R., Dutz, S., Muller, R., and Zeisberger, M. (2006). Magnetic particle hyperthermia: nanoparticle magnetism and materials development for cancer therapy. J. Phys. Condens. Matter. 18, 2919-2934.

Hong, R., Fischer, N.O., Emrick, T., and Rotello, V.M. (2005). Surface PEGylation and ligand exchange chemistry of FePt nanoparticles for biological applications. Chem. Mater. 17, 4617-4621.

Hyeon, T. (2003). Chemical synthesis of magnetic nanoparticles. Chem. Commun. 927-934.

Jana, N.R., Chen, Y., and Peng, X. (2004). Size- and shape-controlled magnetic (Cr, Mn, Fe, Co, Ni) oxide nanocrystals via a simple and general approach. Chem. Mater. 16, 3931-3935.

Judenhofer, M.S., Wehrl, H.F., Newport, D.F., Catana, C., Siegel, S.B., Becker, M., Thielscher, A., Kneilling, M., Lichy, M.P., Eichner, M., et al. (2008). Simultaneous PET-MRI: a new approach for functional and morphological imaging. Nat. Med. 14, 459-465.

Jun, Y.W. Huh, Y.M. Choi, J.S., Lee, J.H., Song, H.T., Kim, S. Yoon, S., Kim, K.S., Shin, J.S., Suh, J.S., et al. (2005). Nanoscale size effect of nanocrystals and their utilization for cancer diagnosis via magnetic resonance imaging (MRI). J. Am. Chem. Soc. 127, 5732-5733.

Jun, Y.W., Choi, J., and Cheon, J. (2007). Heterostructured magnetic nanoparticles: their versatility and high performance capa- bilities. Chem. Commun. 1203-1214.

Jun, Y.W., Seo, J.W., and Cheon, J. (2008a). Nanoscaling laws of magnetic nanoparticles and their applicabilities in biomedical sciences. Acc. Chem. Res. 41, 179-189.

Jun, Y.W., Lee, J.H., and Cheon, J. (2008b). Chemical design of nanoparticle probes for high-performance magnetic resonance imaging. Angew. Chem. Int. Ed. Engl. 47, 5122-5135.

Jung, C.W., and Jacobs, P. (1995). Physical and chemical-properties of superparamagnetic iron-oxide MR contrast agents - ferumoxides, ferumoxtran, ferumoxsil. Magn. Reson. Imaging 13, 661-674.

Kelly, K.L., Coronado, E., Zhao, L.L., and Schatz, G.C. (2003). The optical properties of metal nanoparticles: the influence of size, shape, and dielectric environment. J. Phys. Chem. B 107, 668677.

Kim, J., Piao, Y., and Hyeon, T. (2009). Multifunctional nanostructured materials for multimodal imaging, and simultaneous imaging and therapy. Chem. Soc. Rev. 38, 372-390.

Kubaska, S., Sahani, D.V., Saini, S., Hahn, P.F., and Halpern, E. (2001). Dual contrast enhanced magnetic resonance imaging of the liver with superparamagnetic iron oxide followed by gadolinium for lesion detection and characterization. Clin. Radiol. 56, 410-415.

Lai, C.Y., Trewyn, B.G., Jeftinija, D.M., Jeftinija, K., Xu, S., Jeftinija, S., and Lin, V.S. (2003). A mesoporous silica nanosphere-based carrier system with chemically removable CdS nanoparticle caps for stimuli-responsive controlled release of neurotransmitters and drug molecules. J. Am. Chem. Soc. 125, 4451-4459.

Latham, A.H., and Williams, M.E. (2006). Versatile routes toward functional, water-soluble nanoparticles via trifluoroethylesterPEG-thiol ligands. Langmuir 22, 4319-4326.

Latham, A.H., and Williams, M.E. (2008). Controlling transport and chemical functionality of magnetic nanoparticles. Acc. Chem. Res. 41, 411-420.

Lauffer, R.E. (1987). Paramagnetic metal-complexes as water proton relaxation agents for $\mathrm{nmr}$ imaging - theory and design. Chem. Rev. 87, 901-927.

Lee, J.H., Jun, Y.W., Yeon, S.I., Shin, J.S., and Cheon, J. (2006). Dual-mode nanoparticle probes for high-performance magnetic resonance and fluorescence imaging of neuroblastoma. Angew. Chem. Int. Ed. Engl. 118, 8340-8342.

Lee, J.H., Huh, Y.M., Jun, Y.W., Seo, J.W., Jang, J.T., Song, H.T., Kim, S., Cho, E.J., Yoon, H.G., Suh, J.S., et al. (2007). Artificially engineered magnetic nanoparticles for ultra-sensitive molecular imaging. Nat. Med. 13, 95-99.

Lee, J.H., Lee, K., Moon, S.H., Lee, Y., Park, T.G., and Cheon, J. (2009). All-in-one target-cell-specific magnetic nanoparticles for simultaneous molecular imaging and siRNA delivery. Angew. Chem. Int. Ed. Engl. 121, 4238-4243.

Lee, J.E., Lee, N., Kim, H., Kim, J., Choi, S.H., Kim, J.H., Kim, T., Song, I.C. Park, S.P. Moon, W.K. et al. (2010). Uniform mesoporous dye-doped silica nanoparticles decorated with multiple magnetite nanocrystals for simultaneous enhanced magnetic resonance imaging, fluorescence imaging, and drug delivery. J. Am. Chem. Soc. 132, 552-557.

Lee, J.H., Jang, J.T., Choi, J.S., Moon, S.H., Noh, S.H., Kim, J.W., Kim, J.G., Kim, I.S., Park, K.I., and Cheon, J. (2011). Exchangecoupled magnetic nanoparticles for efficient heat induction. Nat. Nanotechnol. 6, 418-422.

Lee, N., Cho, H.R., Oh, M.H., Lee, S.H., Kim, K., Kim, B.H., Shin, K., Ahn, T.Y., Choi, J.W., Kim, Y.W., et al. (2012). Multifunctional $\mathrm{Fe}_{3} \mathrm{O}_{4} / \mathrm{TaOx}$ core/shell nanoparticles for simultaneous magnetic resonance imaging and X-ray computed tomography. J. Am. Chem. Soc. 134, 10309-10312.

Lewin, M., Carlesso, N., Tung, C.H., Tang, X.W., Cory, D., Scadden, D.T., and Weissleder, R. (2000). Tat peptide-derivatized magnetic nanoparticles allow in vivo tracking and recovery of progenitor cells. Nat. Biotechnol. 18, 410-414.

Ling, Y., Pong, T., Vassiliou, C.C., Huang, P.L., and Cima, M.J. (2011). Implantable magnetic relaxation sensors measure cumulative exposure to cardiac biomarkers. Nat. Biotechnol. 29, 273-278.

Link, S., and El-Sayed, M.A. (1999). Spectral properties and relaxation dynamics of surface plasmon electronic oscillations in gold and silver nanodots and nanorods. J. Phys. Chem. B. 103, 84108426.

Liu, T.Y., Hu, S.H., Liu, D.M., Chen, S.Y., and Chen, I.W. (2009). 
Biomedical nanoparticle carriers with combined thermal and magnetic responses. Nano Today 4, 52-65.

Maccioni, F., Bruni, A., Viscido, A., Colaiacomo, M.C., Cocco, A., Montesani, C., Caprilli, R., and Marini, M. (2006). MR imaging in patents with Crohn disease: Value of T2-versus T1-weighted gadolinium-enhanced MR sequences with use of an oral superparamagnetic contrast agent. Radiology 238, 517-530.

Maier-Hauff, K., Ulrich, F., Nestler, D., Niehoff, H., Wust, P., Thiesen, B., Orawa, H., Budach, V., and Jordan, A. (2011). Efficacy and safety of intratumoral thermotherapy using magnetic ironoxide nanoparticles combined with external beam radiotherapy on patients with recurrent glioblastoma multiforme. J. Neurooncol. 103, 317-324.

Massoud, T.F., and Gambhir, S.S. (2003). Molecular imaging in living subjects: seeing fundamental biological processes in a new light. Genes Dev. 17, 545-580.

Medarova, Z., Pham , W., Farrar, C., Petkova , V., and Moore, A. (2007). In vivo imaging of siRNA delivery and silencing in tumors. Nat. Med. 13, 372-377.

Molday, R.S. (1984). US patent 4, 452, 773

Moser, A., Takano, K., Margulies, D.T., Albrecht, M., Sonobe, Y., Ikeda, Y., Sun, S.H., and Fullerton, E.E. (2002). Magnetic recording: advancing into the future. J. Phys. D Appl. Phys. 35, 157167.

Murray, C.B., Sun, S.H., Doyle, H., and Betley, T. (2001). Monodisperse $3 \mathrm{~d}$ transition-metal ( $\mathrm{Co}, \mathrm{Ni}, \mathrm{Fe}$ ) nanoparticles and their assembly into nanoparticle superlattices. MRS Bull. 26, 985-991.

$\mathrm{Na}$, H.B., Song, I.C., and Hyeon, T. (2009). Inorganic nanoparticles for MRI contrast agents. Adv. Mater. 21, 2133-2148.

Neuberger, T., Schopf, B., Hofmann, H., Hofmann, M., and von Rechenberg, B. (2005). Superparamagnetic nanoparticles for biomedical applications: possibilities and limitations of a new drug delivery system. J. Magn. Magn. Mater. 293, 483-496.

Noh, S.H., Na, W., Jang, J.T., Lee, J.H., Lee, E.J., Moon, S.H., Lim, Y., Shin, J.S., and Cheon, J. (2012). Nanoscale magnetism control via surface and exchange anisotropy for optimized ferrimagnetic hysteresis. Nano Lett. 12, 3716-3721.

Ntziachristos, V., Yodh, A.G., Schnall, M., and Chance, B. (2000). Concurrent MRI and diffuse optical tomography of breast after indocyanine green enhancement. Proc. Natl. Acad. Sci. USA 97, 2767-2722.

Oh, M.H., Lee, N., Kim, H., Park, S.P., Piao, Y., Lee, J., Jun, S.W., Moon, W.K., Choi, S.H., and Hyeon, T. (2011). Large-scale synthesis of bioinert tantalum oxide nanoparticles for X-ray computed tomography imaging and bimodal image-guided sentinel lymph node mapping. J. Am. Chem. Soc. 133, 5508-5515.

O'Neill, K.L., Fairbairn, D.W., Smith, M.J., and Poe, B.S. (1998). Critical parameters influencing hyperthermia-induced apoptosis in human lymphoid cell lines. Apoptosis 3, 369-375.

Ow, H., Larson, D.R., Srivastava, M., Baird, B.A., Webb, W.W., and Wiesner, U. (2005). Bright and stable core-shell fluorescent silica nanoparticles. Nano Lett. 5, 113-117.

Pankhurst, Q.A., Connolly, J., Jones, S.K., and Dobson, J. (2003). Applications of magnetic nanoparticles in biomedicine. J. Phys. D Appl. Phys. 36, 167-181.

Pannaparayil, T., and Komarneni, S. (1989). Synthesis and characterization of ultrafine cobalt ferrites. IEEE Trans. Magn. 25, 4233-4235.

Peer, D., Karp, J.M., Hong, S., FaroKHzad, O.C., Margalit, R., and Langer, R. (2007). Nanocarriers as an emerging platform for cancer therapy. Nat. Nanotech. 2, 751-760.

Peng, S., Wang, C., Xie, J., and Sun, S. (2006). Synthesis and stabilization of monodisperse Fe nanoparticles. J. Am. Chem. Soc. 128, 10676-10677.

Perez, J.M., O'Loughin, T., Simeone, F.J., Weissleder, R., and Josephson, L. (2002a). DNA-based magnetic nanoparticle assembly acts as a magnetic relaxation nanoswitch allowing screening of DNA-cleaving agents. J. Am. Chem. Soc. 124, 2856-2857.

Perez, J.M., Josephson, L., O'Loughlin, T., Högemann, D., and Weissleder, R. (2002b). Magnetic relaxation switches capable of sensing molecular interactions. Nat. Biotechnol. 20, 816-820.

Prodan, E., Radloff, C., Halas, N.J., and Nordlander, P. (2003). A hybridization model for the plasmon response of complex nanostructures. Science 302, 419-422.

Reimer, P., and Balzer, T. (2003). Ferucarbotran (Resovist): a new clinically approved RES-specific contrast agent for contrastenhanced MRI of the liver: properties, clinical development, and applications. Eur. Radiol. 13, 1266-1276.

Reimer, P., Rummeny, E.J., Daldrup, H.E., Balzer, T., Tombach, B., Berns, T., and Peters, P.E. (1995). Clinical-result with resovist A phase-2 clinical-trail. Radiology 195, 489-496.

Rodriguez, O., Fricke, S., Chien, C., Dettin, L., VanMeter, J., Shapiro, E., Dai, H.N., Casimiro, M., lleva, L., Dagata, J., et al. (2006). Contrast-enhanced in vivo imaging of breast and prostate cancer cells by MRI. Cell Cycle 5, 113-119.

Sarikaya, M., Tamerler, C., Jen, A., Schulten, K., and Baneyx, F. (2003). Molecular biomimetics: nanotechnology through biology. Nat. Mater. 2, 577-585

Smith, A.M., and Nie, S. (2010). Semiconductor nanocrystals: structure, properties, and band gap engineering. Acc. Chem. Res. 43, 190-200.

Sun, S.H. (2006). Recent advances in chemical synthesis, selfassembly, and applications of FePt nanoparticles. Adv. Mater 18, 393-403.

Sun, S.H., Zeng, H., Robinson, D.B., Raoux, S., Rice, P.M., Wang, S.X., and Li, G.X. (2004). Monodisperse $\mathrm{MFe}_{2} \mathrm{O}_{4}(\mathrm{M}=\mathrm{Fe}, \mathrm{Co}$, $\mathrm{Mn}$ ) nanoparticles. J. Am. Chem. Soc. 126, 273-279.

Thomas, C.R., Ferris, D.P., Lee, J.H., Choi, E., Cho, M.H., Kim E.S., Stoddart, J.F., Shin, J.S., Cheon, J., and Zink, J.I. (2010). Noninvasive remote-controlled release of drug molecules in vitro using magnetic actuation of mechanized nanoparticles. J. Am. Chem. Soc. 132, 10623-10625.

Tong, S., Hou, S., Zheng, Z., Zhou, J., and Bao, G. (2010). Coating optimization of superparamagnetic iron oxide nanoparticles for high T2 relaxivity. Nano Lett. 10, 4607-4613.

Valberg, P.A., and Feldman, H.A. (1987). Magnetic particle motions within living cells - physical theory and techniques. Biophys. J. $52,551-569$

Wang, J. (2005). Nanomaterial-based amplified transduction of biomolecular interactions. Small 1, 1036-1043.

Wang, N., Butler, J.P., and Ingber, D.E. (1993). Mechanotransduction across the cell-surface and through the cytoskeleton. Science 260, 1124-1127.

Weissleder, R., Moore, A., Mahmood, U., Bhorade, R., Benveniste, H., Chiocca, E.A., and Basilion, J.P. (2000). In vivo magnetic resonance imaging of transgene expression. Nat. Med. 6, 351-354.

Wust, P., Hildebrandt, B., and Sreenivasa, G. (2002). Hyperthermia in combined treatment of cancer. Lancet Oncol. 3, 487-497.

Yi, D.K., Lee, S.S., Papaefthymiou, G.C., and Ying, J.Y. (2006). Nanoparticle architectures templated by $\mathrm{SiO}_{2} / \mathrm{Fe}_{2} \mathrm{O}_{3}$ nanocomposites. Chem. Mater. 18,614-619.

Yoo, D., Lee, J.H., Shin, T.H., and Cheon, J. (2011). Theranostic magnetic nanoparticles. Acc. Chem. Res. 44, 863-874.

Yoo, D., Jeong, H., Preihs, C., Choi, J.S., Shin, T.H., Sessler, J.L., and Cheon, J. (2012). Double-effector nanoparticles: a synergistic approach to apoptotic hyperthermia. Angew. Chem. Int. Ed. Engl. 51, 12482-12485

Yu, M.K., Jeong, Y.Y., Park, J., Park, S., Kim, J.W., Min, J.J., Kim, K., and Jon, S. (2008). Drug-loaded superparamagnetic iron oxide nanoparticles for combined cancer imaging and therapy in vivo. Angew. Chem. Int. Ed. Engl. 47, 5362-5365.

Zhang, Y., Kohler, N., and Zhang, M. (2002). Surface modification of superparamagnetic magnetite nanoparticles and their intracellular uptake. Biomaterials 23, 1553-1561. 